\title{
S100ß, Matrix Metalloproteinase-9, D-dimer, and Heat Shock Protein 70 Are Serologic Biomarkers of Acute Cerebral Infarction in a Mouse Model of Transient MCA Occlusion
}

\author{
Jong-Il Choi, M.D., Ph.D., ${ }^{1}$ Sung-Kon Ha, M.D., Ph.D., ${ }^{2}$ Dong-Jun Lim, M.D., Ph.D., ${ }^{2}$ Sang-Dae Kim, M.D., Ph.D., ${ }^{2}$ \\ Se-Hoon Kim, M.D., Ph.D. ${ }^{2}$ \\ Department of Neurosurgery, Hallym University Kangnam Sacred Heart Hospital, Hallym University College of Medicine, Seoul, Korea \\ Department of Neurosurgery, Korea University Medical Center, Seoul, Korea
}

Objective : Diagnosing acute cerebral infarction is crucial in determining prognosis of stroke patients. Although many serologic tests for prompt diagnosis are available, the clinical application of serologic tests is currently limited. We investigated whether S100ß, matrix metalloproteinase-9 (MMP-9), D-dimer, and heat shock protein 70 (HSP70) can be used as biomarkers for acute cerebral infarction.

Methods : Focal cerebral ischemia was induced using the modified intraluminal filament technique. Mice were randomly assigned to 30-minute occlusion $(n=10), 60$-minute occlusion $(n=10)$, or sham $(n=5)$ groups. Four hours later, neurological deficits were evaluated and blood samples were obtained. Infarction volumes were calculated and plasma S100ß, MMP-9, D-dimer, and HSP70 levels were measured using enzyme-linked immunosorbent assay.

Results : The average infarction volume was $12.32 \pm 2.31 \mathrm{~mm}^{3}$ and $46.9 \pm 7.43 \mathrm{~mm}^{3}$ in the 30 - and 60 -minute groups, respectively. The mean neurological score in the two ischemic groups was $1.6 \pm 0.55$ and $3.2 \pm 0.70$, respectively. S100ß, MMP-9, and HSP70 expressions significantly increased after 4 hours of ischemia $(p=0.001)$. Furthermore, S100 $\beta$ and MMP-9 expressions correlated with infarction volumes $(p<0.001)$ and neurological deficits $(p<0.001)$. There was no significant difference in $\mathrm{D}$-dimer expression between groups $(p=0.843)$. The area under the receiver operating characteristic curve (AUC) showed high sensitivity and specificity for MMP-9, HSP70 (AUC=1), and S100ß (AUC=0.98).

Conclusion : $\mathrm{S100 \beta}, \mathrm{MMP}-9$, and HSP70 can complement current diagnostic tools to assess cerebral infarction, suggesting their use as potential biomarkers for acute cerebral infarction.

Key Words : Acute ischemic stroke · Biomarkers · Cerebral infarction volume.

\footnotetext{
- Received : June 27, 2017 •Revised : September 8, 2017 •Accepted : October 8, 2017

- Address for reprints : Sung-Kon Ha, M.D., Ph.D.

Department of Neurosurgery, Korea University Ansan Hospital, 123 Jeokgeum-ro, Danwon-gu, Ansan 15355, Korea Tel : +82-31-412-5050, Fax : +82-31-412-5054, E-mail : sungkha@yahoo.com

This is an Open Access article distributed under the terms of the Creative Commons Attribution Non-Commercial License (http://creativecommons.org/licenses/by-nc/4.0) which permits unrestricted non-commercial use, distribution, and reproduction in any medium, provided the original work is properly cited.
} 


\section{INTRODUCTION}

The most widely used diagnostic tool for acute cerebral infarction is neuroimaging, which includes techniques such as computed tomography (CT) and magnetic resonance imaging. Diffusion weighted imaging has $>90 \%$ sensitivity for diagnosing acute cerebral infarction, in particular ${ }^{10)}$. In addition, currently many efforts are underway to enhance the diagnostic ability of neuroimaging tools; a study on biomarkers in acute cerebral infarctions is one example.

Biomarkers have shown efficacy in other medical fields, such as acute coronary artery syndrome, and can provide a great deal of information to assist with early diagnosis and intervention. This suggests that biomarkers can facilitate early therapeutic interventions when combined with neurological examination and neuroimaging in acute cerebral infarction $^{25,26)}$

Many studies have indicated that biomarkers linked to the ischemic cascade have a potential role in assessing acute cerebral infarction, such as early neurologic deterioration, final infarct volume, clinical status, and functional outcome ${ }^{5,9)}$. However, to the best of our knowledge, no study has discovered a successful clinical biomarker. This may be because most proteins that increase during acute cerebral infarction do not have the major features that are required to be a significant and useful biomarker. These characteristics are : 1) brain specificity; 2) presence in plasma to enable blood, rather than cerebrospinal fluid, sampling; 3) presence in plasma samples at an early stage of onset of clinical manifestations; 4) expression that corresponds to the size of the lesion; and 5) prognostic value. Currently, the use of biomarkers for acute cerebral infarction has been confined to research laboratories owing to these constraints, and none has been used in a clinical setting ${ }^{18)}$.

To overcome these difficulties and enhance their diagnostic utility, studies have attempted to combine several biomarkers for use in a panel. Reynolds et al. ${ }^{19)}$ and Lynch et al. ${ }^{16)}$ have shown that a panel of biomarkers can be cost-effective and time-saving in combination with brain imaging. This allows physicians to promptly identify subsets of patients who may benefit from immediate intervention and critical care.

There is debate regarding whether a panel of biomarkers can be clinically useful, have a good predictive rate, and aid pre-existing diagnostic tools ${ }^{15)}$. Moreover, a limited number of biomarker studies have reported results during the early stages of acute cerebral infarction. This time-point is the critical window for treatment using intravenous recombinant tissue plasminogen activator (IV-tPA) within 4.5 hours, or interventional treatment options within 6 hours of stroke onset ${ }^{3)}$.

To create a panel of biomarkers that can be used during the acute stage of cerebral infarction, it is essential to discover and select biomarkers that can be detected in the plasma at the point of ischemic onset, and that are specific to ischemic brain injury. In this study, we selected $\mathrm{S} 100 \beta$, matrix metalloproteinase-9 (MMP-9), D-dimer, and heat shock protein 70 (HSP70) from the proteins that are involved in various pathological processes during acute cerebral infarction, such as brain-specific markers, inflammatory mediators, thrombosis/ hemostasis-related markers, and neuroprotective agents, to test their efficacy as clinically applicable biomarkers ${ }^{15,16)}$.

We used a mouse model of infarction with transient middle cerebral artery (MCA) occlusion to investigate the use of these proteins as biomarkers in acute cerebral infarction within 4.5 hours of symptom onset, the time when IV-tPA administration is feasible.

\section{MATERIALS AND METHODS}

\section{Focal cerebral infarction in mice}

Twenty-five C57BL/6 adult male mice (age, 7-8 weeks) weighing 23-25 g, were used in this study. The Institutional Animal Care and Use Committee reviewed and approved the animal protocols that were used herein. Mice were randomly assigned to three experimental groups : sham-operation $(n=5)$, 30-minute middle cerebral artery occlusion (MCAO; $n=10$ ), and 60-minute MCAO $(\mathrm{n}=10)$.

Focal cerebral ischemia was induced by transiently occluding the MCA in these mice using a modified intraluminal filament technique ${ }^{29)}$. Mice were anesthetized with 5\% isoflurane in $30 \% \mathrm{O}_{2} / 70 \% \mathrm{~N}_{2} \mathrm{O}$ using the $\mathrm{V}-10$ Anesthesia system (VetEquip, Inc., Pleasanton, CA, USA). Following induction of anesthesia, the level of isoflurane was reduced and maintained at $1.5 \%$. After a midline neck incision, the left common and external carotid arteries were isolated and ligated, and a microvascular clip was temporarily placed on the left internal carotid artery.

An 8-0 nylon monofilament coated with silicon resin was 
introduced through the incision into the common carotid artery and advanced $9 \mathrm{~mm}$ distal to the carotid bifurcation to achieve the MCAO. The nylon monofilament was removed after 30 or 60 minutes for mice in the 30- or 60-minute MCAO groups, respectively. Mice in the sham-operation group received a midline neck incision with left common and external carotid artery isolation and ligation, but did not undergo any further manipulation. The rectal temperature was maintained at $37 \pm 0.2^{\circ} \mathrm{C}$ throughout the surgical procedure. After suturing the skin incision, the mice were returned to their cages.

\section{Neurological scores}

We evaluated the neurological deficits in each group 4 hours after the removal of the nylon filament. Mice from each group underwent behavioral assessment using a 6-point deficit score ${ }^{11)}$.

Scoring indications were as follows : 0, normal motor function; 1 , mild circling behavior with or without inconsistent rotation when picked up by the tail and with $<50 \%$ attempts to rotate to the contralateral side; 2 , mild consistent circling with $>50 \%$ attempts to rotate to the contralateral side; 3 , consistent strong and immediate circling with the mouse maintaining a rotating position for more than 1-2 seconds, with its nose almost reaching its tail; 4 , severe rotation progressing into barreling, loss of walking, or a righting reflex; and 5, a comatose or moribund state.

\section{Measurement of cerebral infarction volume}

Brains were extracted to identify the presence of infarction. Infarct volumes were measured in all mice. The brains were removed and sliced coronally into four 2-mm slices with a Brain Matrix (Braintree Scientific, Braintree, MA, USA) on ice. Slices were incubated in 2\% 2, 3, 5-triphenyltetrazolium chloride and warmed to room temperature for 20 minutes, followed by fixation with $4 \%$ paraformaldehyde at $4^{\circ} \mathrm{C}$ for 48 hours before scanning. The infarct area of each section was measured using National Institutes of Health (NIH) Image J software version 9 (National Institutes of Health, Bethesda, MD, USA), and the total infarct volume was calculated.

Ipsilateral and contralateral hemisphere volumes were also determined to correct for cerebral edema, which can artificially affect infarct volume ${ }^{23)}$. The following formula was used to calculate the edema-corrected infarct volume : corrected infarct volume $=$ contralateral non-infarct volume-ipsilateral non-infarct volume $e^{24)}$.

Assessment of plasma biomarkers (S100 $\beta$, MMP9, D-dimer, and HSP70) using enzyme-linked immunosorbent assay (ELISA)

For quantitative analyses of plasma S100 $\beta$, MMP-9, D-dimer, and HSP70 expression, blood samples were obtained by cardiac puncture with a 1-mL syringe and 22-gauge needle 4 hours post-MCAO. Blood samples were collected in glass tubes with ethylenediaminetetraacetic acid, centrifuged at $1000 \mathrm{~g}$ for 30 minutes, and supernatants were frozen at $-80^{\circ} \mathrm{C}$ until further analysis. The amount of plasma necessary for each assay was calculated, and the optimal dilution ratios were determined (S100 $\beta$, no dilution; MMP-9, $1: 20$; D-dimer, $1: 4$; HSP70, no dilution).

The plasma concentrations of S100 $\beta$, MMP-9, D-dimer, and HSP70 were calculated using mouse protein S100 $\beta$ (CSB-EL0206643MO; CUSABIO, Wuhan, China), mouse total MMP9 (MMPT90; R\&D Systems, Minneapolis, MN, USA), mouse D-dimer (E03D0224; BlueGene, Shanghai, China), and mouse HSP70 (CSB-E08311m; CUSABIO) ELISA kits. All sandwich ELISAs were performed using standard procedures according to the manufacturer's instructions.

\section{Sensitivity and specificity of the biomarkers}

To evaluate the sensitivity and specificity of the biomarkers, we constructed a receiver operating characteristic (ROC) curve, which plotted test sensitivity as the y coordinate, and 1-specificity, or the false positive rate, as the $\mathrm{x}$ coordinate. We estimated the area under the ROC curve (AUC), which is measured between $0-1$, since both axes have values ranging from $0-1$. The best overall diagnostic performance was indicated by an AUC value of 1 .

\section{Statistical analysis}

Statistical analyses were performed using the Statistical Package for the Social Sciences version 18.0 for Windows (SPSS Corp., Chicago, IL, USA). The Kruskal-Wallis and Mann-Whitney U tests were used to compare the distributions of continuous variables between groups. Two-sided hypothetical tests were used. The Spearman correlation coefficient was used to analyze the relationship between the biomarkers, infarct volumes, and neurological scores. A $p$ value $<0.05$ was considered statistically significant. 


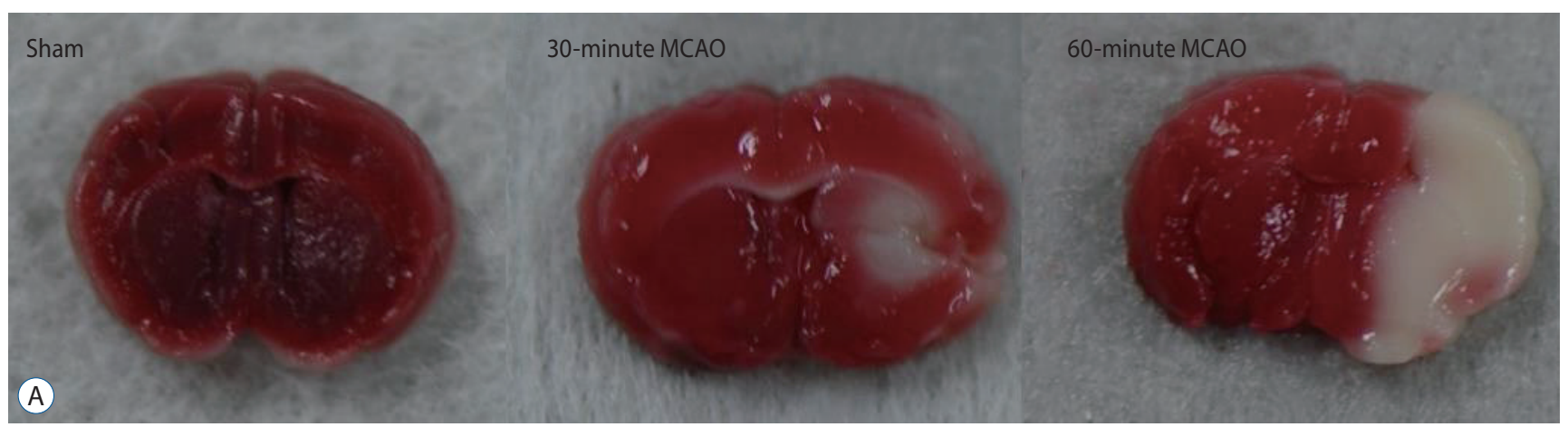

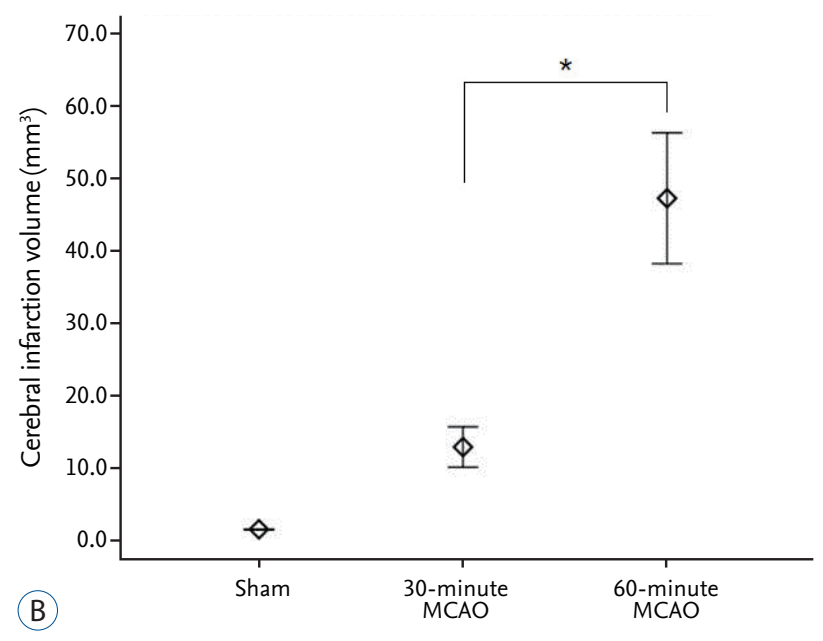

\section{RESULTS}

\section{Cerebral infarction volume}

Cerebral infarcts were found in the hemisphere ipsilateral to the occlusion in all mice that were exposed to transient ischemia (30-minute MCAO, $\mathrm{n}=10 ; 60$-minute MCAO, $\mathrm{n}=10$ ). No infarction was noted in mice from the sham group $(n=5$; Fig. 1A). The volume of cerebral infarction increased with MCA occlusion time. The average infarct volume was $12.32 \pm 2.31$ $\mathrm{mm}^{3}$ in the 30-minute occlusion group, and infarcts were mainly confined to the basal ganglia. The average infarct volume was $46.9 \pm 7.43 \mathrm{~mm}^{3}$ in the 60 -minute occlusion group, and the distribution was more extensive, affecting an area from the basal ganglia to the cerebral cortex. A significant difference in infarct volume was found between the 30-minute and 60-minute MCAO groups ( $p=0.008$ ), with greater infarct volume in the 60-minute ischemia group (Fig. 1B).
Fig. 1. A : Coronal brain sections. Infarcts are visualized as unstained regions following 2, 3, 5-triphenyltetrazolium chloride staining. No infarct is seen in sham-operated mice. Infarcts are confined to the basal ganglia in 30-minute MCAO mice, and extend from the basal ganglia to the cerebral cortex in the 60-minute MCAO mice. B : Comparison of the infarction volumes between the sham-operated, 30-minute MCAO, and 60 -minute MCAO groups. The infarction volume is significantly larger in the 60-minute MCAO group when compared with the 30-minute group $(p=0.008)$. Data are expressed as mean \pm standard deviation. ${ }^{*} p<0.05$. MCAO : middle cerebral artery occlusion.

\section{Neurological score}

Neurological deficits were found in all mice that underwent transient ischemia (30-minute MCAO, $\mathrm{n}=10 ; 60$-minute MCAO, $\mathrm{n}=10$ ). No neurological deficit was noted in mice from the sham group $(n=5)$. The average neurological scores in the 30-minute and 60-minute ischemia groups were 1.6 \pm 0.55 and $3.2 \pm 0.70$, respectively. The neurological score of the sham group was 0 . A statistical comparison of the neurological scores revealed a significant difference between the 30-minute and 60-minute MCAO groups ( $p=0.002$; Fig. 2).

\section{Expression of plasma biomarkers}

\section{$\mathrm{S} 100 \beta$}

Quantitative ELISA measurements of plasma S100 $\beta$ showed average values of $3.9 \pm 2.47 \mathrm{pg} \cdot \mathrm{mL}^{-1}, 16.8 \pm 8.34 \mathrm{pg} \cdot \mathrm{mL}^{-1}$, and 55.1 $\pm 21.63 \mathrm{pg} \cdot \mathrm{mL}^{-1}$ in the sham, 30 -minute $\mathrm{MCAO}$, and 60 -minute MCAO groups, respectively. Thus, the concentration of $S 100 \beta$ in both infarction groups was significantly increased 


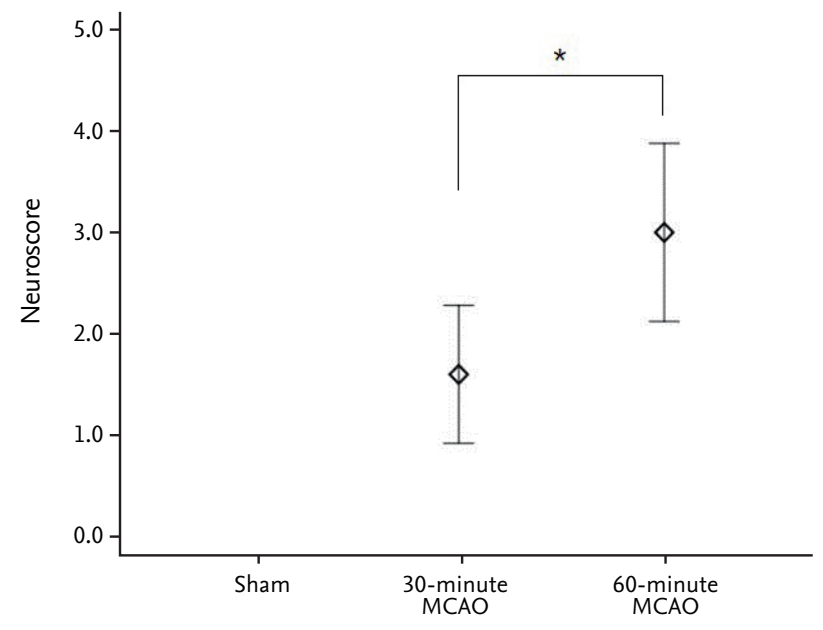

Fig. 2. Comparison of the neurological scores between the shamoperated, 30-minute MCAO, and 60-minute MCAO groups. Shamoperated mice do not show any neurological deficit. The neurological score is significantly higher in the 60-minute group when compared with the 30-minute group ( $p=0.032$ ). Data are expressed as mean \pm standard deviation. ${ }^{*} p<0.05$. MCAO : middle cerebral artery occlusion.

when compared with the sham group ( $p=0.001)$. In addition, the 60-minute occlusion group had a significantly higher $S 100 \beta$ concentration when compared with the 30 -minute group ( $p=0.001$; Fig. $3 \mathrm{~A}$ ). The plasma $S 100 \beta$ concentration was significantly positively correlated with cerebral infarct volume and neurological score, with statistical values of $r=0.867$ $(p<0.001)$ and $\mathrm{r}=0.802(p<0.001)$, respectively (Fig. 3B and C).

\section{MMP-9}

Quantitative ELISA measurements of plasma MMP-9 showed average values of $0.63 \pm 0.09 \mathrm{ng} \cdot \mathrm{mL}^{-1}, 1.20 \pm 0.22 \mathrm{ng} \cdot \mathrm{mL}^{-1}$, and $1.88 \pm 0.41 \mathrm{ng} \cdot \mathrm{mL}^{-1}$ in the sham, 30-minute MCAO, and 60-minute MCAO groups, respectively. MMP-9 concentration in both infarction groups was significantly increased when compared with the sham group $(p=0.001)$. In addition, the 60-minute occlusion group showed a significantly higher MMP-9 concentration than the 30-minute occlusion group ( $p=0.001$; Fig. 4A). The plasma MMP-9 concentration was significantly positively correlated with both cerebral infarct volume and neurological score, with statistical values of $\mathrm{r}=0.890$ $(p<0.001)$ and $\mathrm{r}=0.879(p<0.001)$, respectively (Fig. $4 \mathrm{~B}$ and C).

\section{D-dimer}

Quantitative ELISA measurements of plasma D-dimer showed average values of $3689 \pm 335 \mathrm{pg} \cdot \mathrm{mL}^{-1}, 3884 \pm 532 \mathrm{pg} \cdot \mathrm{mL}^{-1}$,
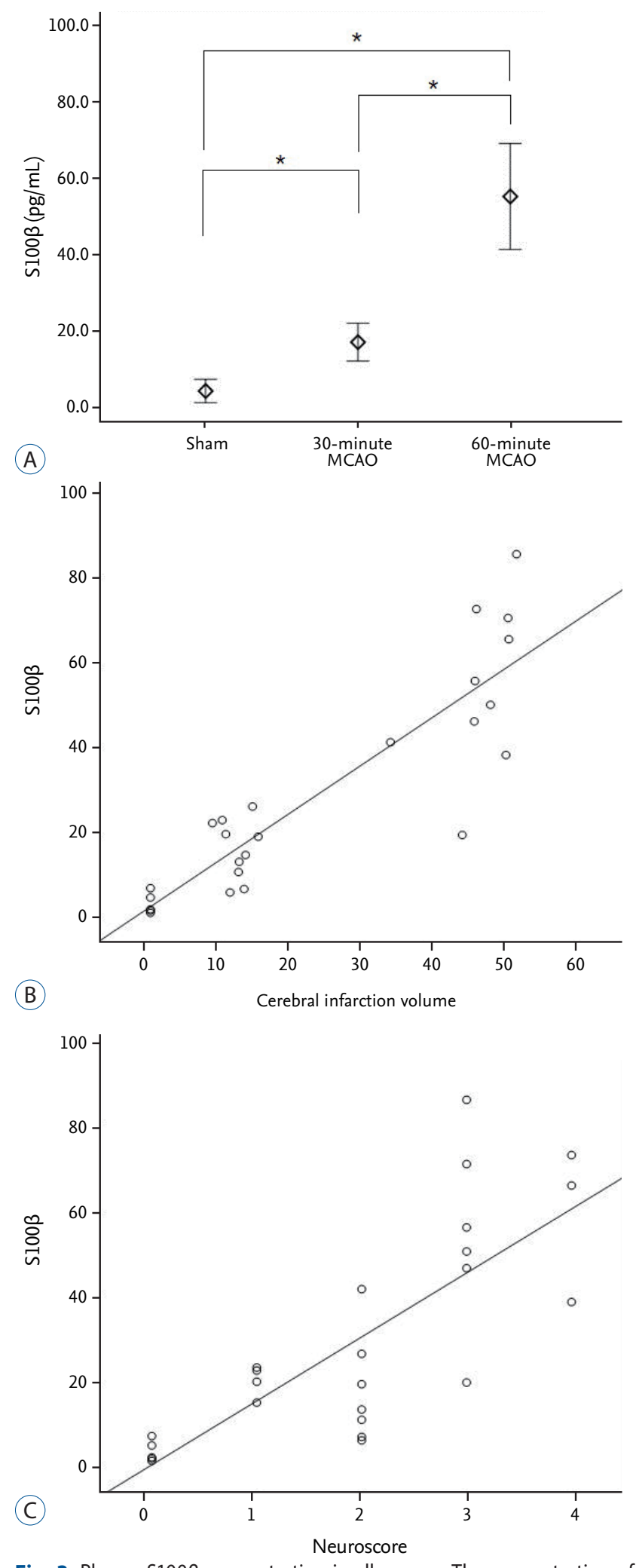

Fig. 3. Plasma $S 100 \beta$ concentration in all groups. The concentration of $S 100 \beta$ increases with occlusion time $(p=0.001 ; A)$ and correlates significantly with infarct volume $(r=0.867, p<0.001 ; B)$ and neurological $\operatorname{deficit}(\mathrm{r}=0.802, p<0.001 ; \mathrm{C}) .{ }^{*} p<0.05$. 

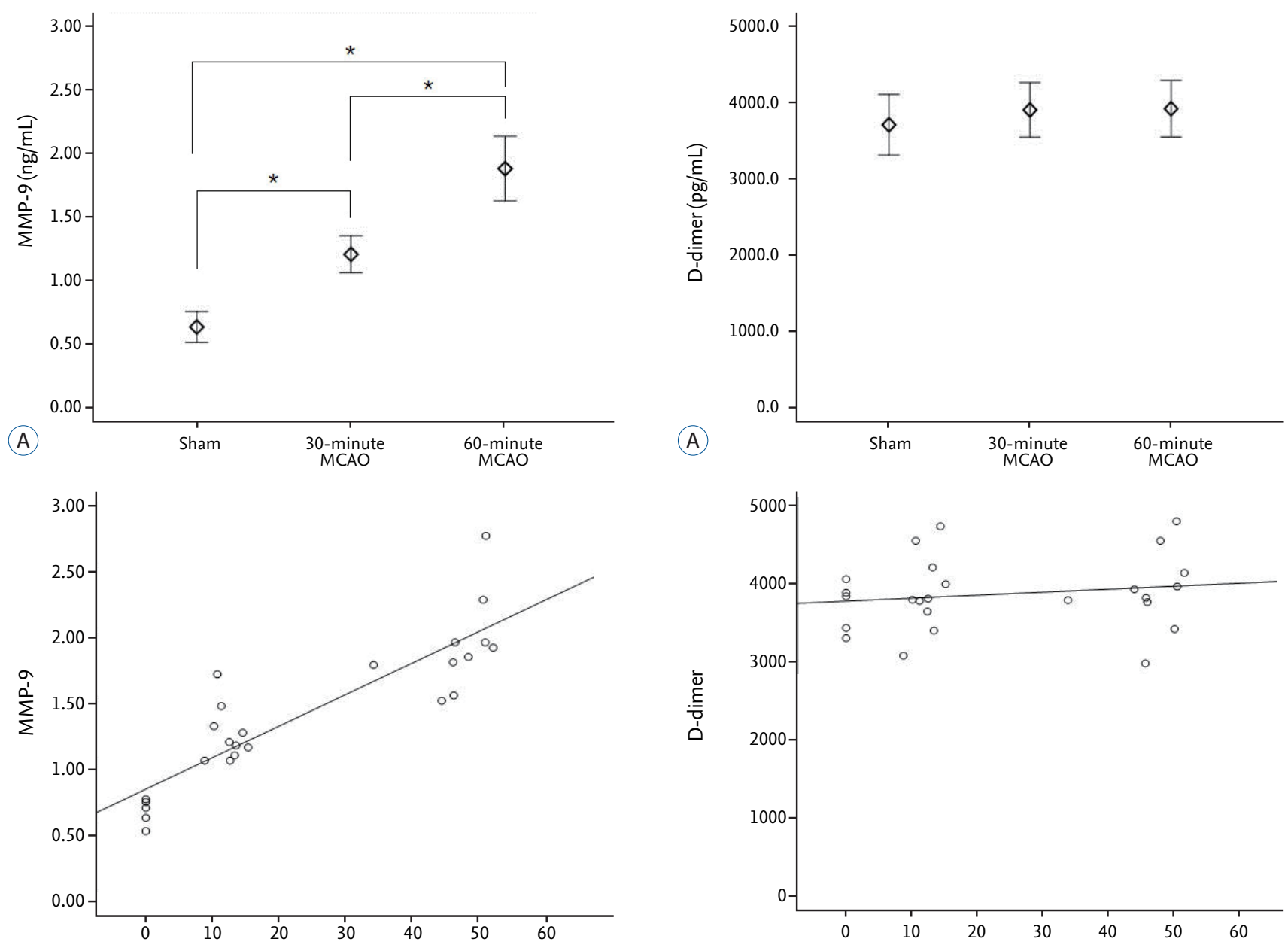

(B)

Cerebral infarction volume

(B)

Cerebral infarction volume

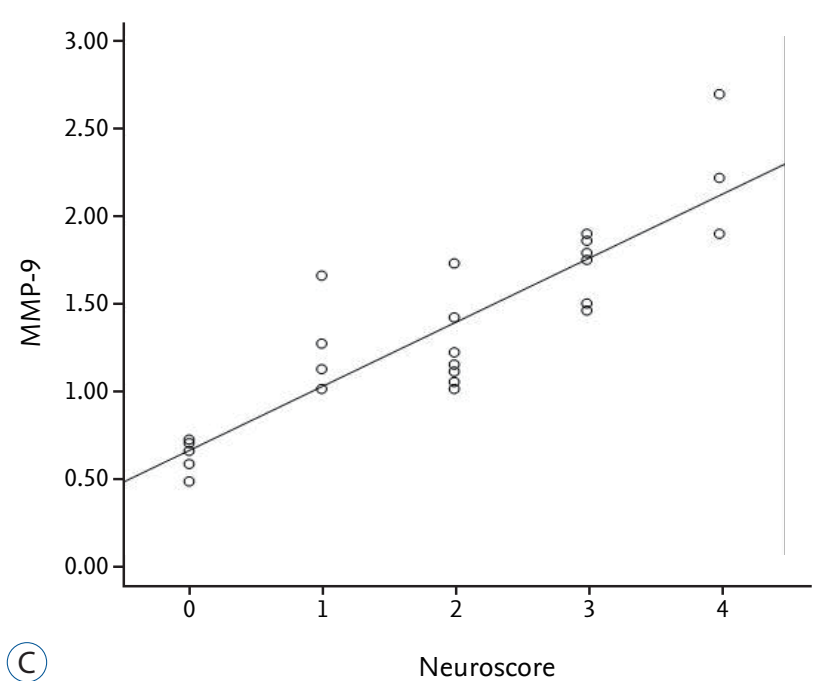

Fig. 4. Plasma MMP-9 concentration in all groups. The concentration of MMP-9 increases with occlusion time $(p=0.001 ; A)$ and correlates significantly with infarct volume $(r=0.890, p<0.001 ; B)$ and neurological deficit $(r=0.879, p<0.001 ; C) .{ }^{*} p<0.05$. MMP-9: matrix metalloproteinase-9.

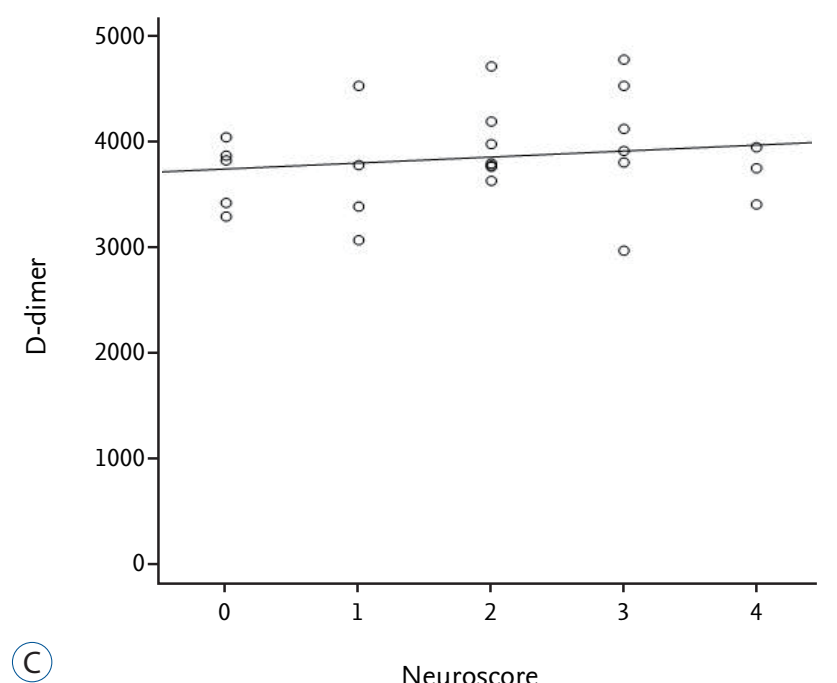

Fig. 5. Plasma D-dimer concentration in all groups. There is no significant difference in $D$-dimer concentration between groups $(p=0.843 ; A)$, and no correlation with infarct volume $(r=0.255, p=0.218 ; B)$ or neurological score $(r=0.139, p=0.507 ; C)$. MCAO : middle cerebral artery occlusion. 
(A)
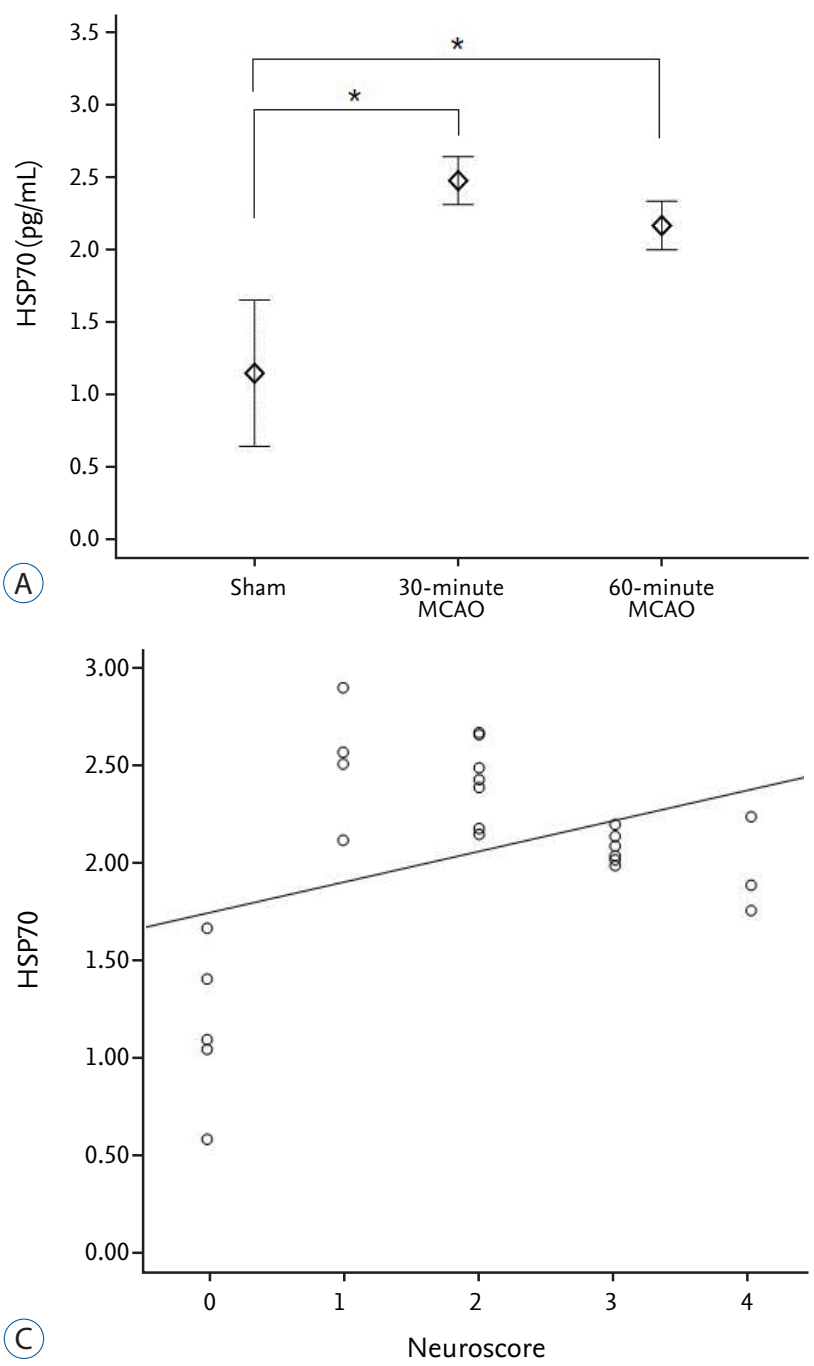

and $3900 \pm 1228 \mathrm{pg} \cdot \mathrm{mL}^{-1}$ in the sham, 30-minute MCAO, and 60-minute MCAO groups, respectively. There was no significant difference in plasma $\mathrm{D}$-dimer concentrations between the three groups ( $p=0.843$; Fig. $5 \mathrm{~A}$ ). In addition, there was no significant correlation with cerebral infarct volume $(r=0.255$, $p=0.218)$ or neurological score $(r=0.139, p=0.507$; Fig. $5 B$ and $C)$.

\section{HSP70}

Quantitative ELISA measurements of plasma HSP70 showed average values of $1.15 \pm 0.41 \mathrm{ng} \cdot \mathrm{mL}^{-1}, 2.48 \pm 0.15 \mathrm{ng} \cdot \mathrm{mL}^{-1}$, and 2.24 $\pm 0.19 \mathrm{ng} \cdot \mathrm{mL}^{-1}$ in the sham, 30-minute $\mathrm{MCAO}$, and 60-minute MCAO groups, respectively. The concentration of HSP70 in both infarction groups was significantly increased when compared with the sham group ( $p=0.001$, for both). In addition, the HSP70 concentration was higher in the 30 -minute occlusion group when compared with the 60-minute occlusion group,

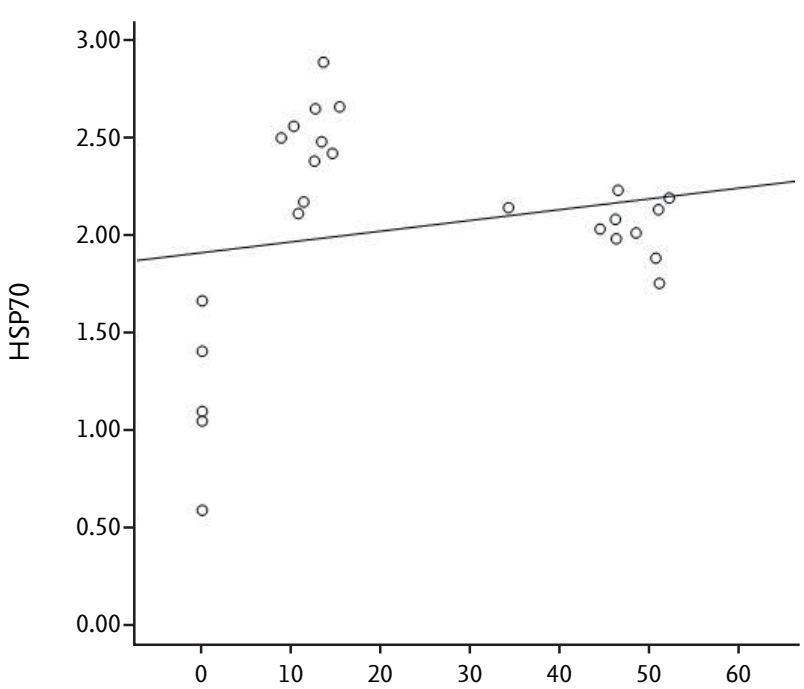

(B)

Cerebral infarction volume

Fig. 6. Plasma HSP70 concentration in all groups. HSP70 concentration increases significantly in both infarction groups $(p=0.001)$ when compared to that of the sham group (A); however, there is no correlation between HSP70 concentration and infarct area $(r=0.174, p=0.406 ; \mathrm{B})$ or neurological score $(r=0.138, p=0.510 ; C) .{ }^{*} p<0.05$. HSP70 : heat shock protein $70, \mathrm{MCAO}$ : middle cerebral artery occlusion.

but this did not reach statistical significance ( $p=0.66$; Fig. 6A). The plasma HSP70 concentration did not correlate with cerebral infarct volume $(\mathrm{r}=0.174, p=0.406)$ or neurological score $(\mathrm{r}=0.138, p=0.510$; Fig. $6 \mathrm{~B}$ and $\mathrm{C})$.

\section{Sensitivity and specificity of the biomarkers}

The area under the ROC curve (AUC) showed high sensitivity and specificity for MMP-9 and HSP70, which both showed an AUC of 1, and for S100 $\beta$, which showed an AUC of 0.98. The AUC for D-dimer was 0.58 (Fig. 7).

\section{DISCUSSION}

The levels of many plasma proteins are elevated in patients with acute ischemic stroke ${ }^{17)}$. These proteins are associated 


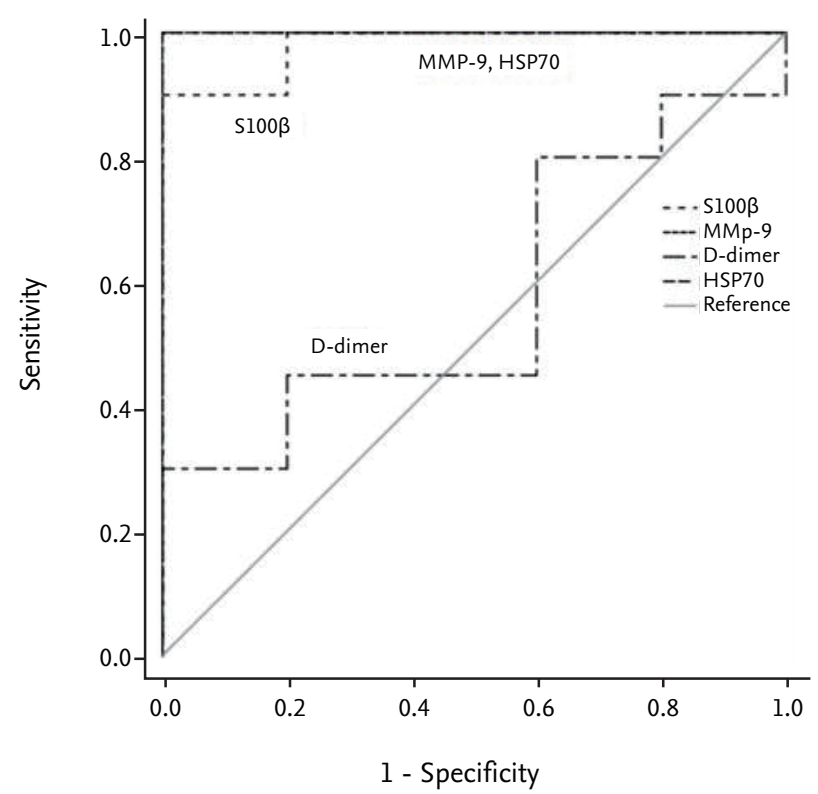

Fig. 7. Sensitivity and specificity of the biomarkers. MMP-9 and HSP70 both have an AUC of 1. S100 $\beta$ has an AUC of 0.98 . The AUC for D-dimer is 0.58 . MMP-9 : matrix metalloproteinase-9, HSP70 : heat shock protein 70 , $A U C$ : the area under the receiver operating characteristic curve.

with certain biochemical changes that are involved in the pathogenesis of acute cerebral infarction.

The early phase of ischemia induces the activation of astroglial cells, neuronal damage, increased oxidative stress, and the release of inflammatory mediators. During the acute ischemic phase, brain-specific proteins are released into the bloodstream through blood-brain barrier (BBB) disruption, allowing for their serological detection. Additionally, the levels of inflammatory mediators and molecules related to thrombosis/hemostasis that are present in the bloodstream become elevated during the acute ischemic phase $\mathrm{e}^{25,26)}$.

Despite theoretical evidence for these changes, no single biomarker has sufficient predictive value to serve as an early diagnostic indicator of ischemic stroke, or to distinguish stroke from other conditions. A recently published meta-analysis on stroke-related serological markers reported that each marker failed to improve the predictive value obtained in current clinical models ${ }^{26)}$. Consequently, recent research has focused on enhancing diagnostic accuracy by establishing a panel of biomarkers to overcome the low sensitivity and specificity of individual biomarkers ${ }^{15,26)}$.

The Biomarker Rapid Assessment in Ischemic Injury Study group evaluated plasma D-dimer, S100 $\beta$, MMP-9, and brain natriuretic peptide levels from patients within 24 hours of ischemic stroke or stoke-mimicking events. The group analyzed the diagnostic prediction rate and reported a diagnostic sensitivity of $90 \%$. In addition, this rate was further increased when combined with $\mathrm{CT}^{15}$. To enhance the diagnostic accuracy and treatment efficacy of acute cerebral infarction, further research into an effective panel of biomarkers is required. To achieve this, highly sensitive and specific biomarkers for acute cerebral infarction need to be identified and selected.

$S 100 \beta$ is a calcium-binding peptide secreted by astrocytes during brain injury, neurodegeneration, and psychiatric disorders. The interaction between $S 100 \beta$ and the cerebral immune system leads to the enhanced survival of developing neurons at low concentrations of $S 100 \beta$. At higher concentrations, S100 $\beta$ stimulates the production of pro-inflammatory cytokines and apoptosis in vitro. Increased serum levels of S100 $\beta$ are found with ischemic stroke and many other acute and chronic conditions, such as traumatic brain injury, Alzheimer' $s$ disease, and schizophrenia ${ }^{14)}$.

In ischemic stroke, the serum concentration of S100 $\beta$ reaches its maximal concentration at days $2-4$. Peak concentrations correlate with higher NIH Stroke Scale scores, infarct sizes, and the clinical course in both infarction and subarachnoid hemorrhage ${ }^{6,7)}$. Reynolds et al. ${ }^{19)}$ reported that $S 100 \beta$ concentration, which peaks within 0-12 hours of cerebral infarction, is highly relevant in ischemia. In addition, this pattern is found within 0-6 hours of stroke. Several other studies have reported a higher S100 $\beta$ concentration within 12 hours, or 6 hours at the earliest, of cerebral infarction onset when compared with that observed in control groups ${ }^{6,15,19,27)}$.

In our study, the concentration of S100 $\beta$ increased as ischemia developed, and further increased following a longer duration of MCAO. There was a positive correlation between S100 $\beta$ concentration and both infarct volume and the degree of neurological deficit. Overall, our results are consistent with previous reports that show that the concentration of plasma S100 $\beta$ can be used to complement traditional early diagnostic tools for acute cerebral infarction and to assess its severity.

MMPs are a family of 14 enzymes, including a subset that is active in brain tissue. MMPs are grouped into stromelysins, gelatinases, collagenases, membrane-type proteinases, and matrilysins ${ }^{19)}$. MMPs are produced in latent form and require activation that leads to their toxicity. Once active, these enzymes are implicated in many different processes, such as dis- 
ruption of the BBB, capillary damage, and prolonged inflammation. Consequently, they promote the secondary progression of ischemia and hemorrhagic transformation ${ }^{4)}$. Both inflammation and alterations to the vascular wall structure due to MMP activity have been implicated in the chronic and progressive damage of intracranial atherosclerosis and acutephase injury ${ }^{2}$.

MMP-9 is widely expressed in brain tissue. It can induce cerebral edema and hemorrhagic transformation via disruption of the BBB, thus increasing its permeability during ischemia. MMP-9 is produced by leukocytes, neurons, glial cells, vascular smooth muscle cells, and endothelial cells. It mediates inflammation by disassembling collagen IV, the basal lamina, and fibronectin. MMP-9 concentrations increase after 2 hours of ischemia, peak at 72 hours, and decrease 120 hours after onset. MMP-9 levels increase rapidly during cerebral infarction in accordance with the size of the infarct; therefore, it is a potential biomarker to aid with the early diagnosis and severity assessment of acute cerebral infarction ${ }^{20)}$.

This study suggests that the concentration of plasma MMP-9 can be used to complement current diagnostic protocols in the detection of early stage acute cerebral infarction and to estimate its severity. We found that the concentration of MMP-9 increased as ischemia developed, and higher concentrations were associated with a longer duration of MCAO. There was also a positive correlation with infarct volume and the degree of neurological deficit.

D-dimer, a marker of plasmin-mediated fibrin degradation, is cross-linked to fibrin degradation products (FDP) and indicates vessel occlusion. Plasmin splits fibrin into FDP and Ddimers when the coagulation and fibrinolytic system is activated. D-dimer is elevated under various conditions such as deep vein thrombosis, pulmonary thromboembolism, myocardial infarction, disseminated intravascular coagulation, surgery, trauma, and stroke ${ }^{1)}$.

An increase in the concentration of D-dimer during ischemic stroke is due to activation of the hemostatic system by meningeal irritation because of increased intracranial pressure, and activation of the coagulation pathway due to brain tissue injury and cerebrovascular disruption ${ }^{8)}$. The plasma concentration of D-dimer increases 24 hours after cerebral infarction; however, we did not find any significant increase in the concentration of D-dimer following MCAO. In addition, it did not correlate with infarct volume or the degree of neuro- logical deficit at the early stage of acute cerebral infarction.

HSP70 is a molecular chaperone induced by various pathological insults, such as hyperthermia, apoptotic stimuli, oxidative stress, and ischemia ${ }^{29)}$. An increase in the gene expression of heat shock proteins, such as HSP70, in the brain is triggered by a variety of insults, including ischemia ${ }^{18)}$. Yenari et al. ${ }^{28)}$ have suggested that increased HSP70 expression indicates cellular stress. Stressful conditions can result in the denaturing of proteins. The presence of these altered proteins triggers the transcription of heat shock proteins, such as HSP70, which bind to the denatured proteins to prevent further denaturation.

During cerebral infarction, HSP70 can be expressed in neurons, astrocytes, microglia, or endothelial cells in the brain ${ }^{18)}$. Over-expression of HSP70 protects these cells against ischemic conditions by preventing protein denaturation, and stabilizing and restoring the functions of partially denatured proteins ${ }^{18)}$. The HSP70 chaperone family regulates both apoptotic and necrotic cell death by blocking apoptosis at several different steps in the pathway. Based on this knowledge, we sought to verify whether HSP70 could serve as a biomarker of the acute stage of cerebral infarction. There was a significant increase in HSP70 following ischemia; however, this increase did not correlate with infarct volume or the degree of neurological deficit in our experimental setting.

As for this result, there is a clinical report implying that HSP 70 expression from lymphocytes correlated with both the severity of the stroke and improvement in neurologic deficit scores after stroke ${ }^{12)}$. Therefore, animal and clinical studies will be need to investigate the relations of gene expressions from infarcted brain tissue and plasma during acute cerebral infarction.

In summary, S100 $\beta$, MMP-9, and HSP70 were significantly increased during the acute stage of cerebral infarction induced by transient MCAO in our mouse model. In addition, the plasma concentration of S100 $\beta$ and MMP-9 correlated with the volume of cerebral infarction and the degree of neurological deficit. This suggests that they may be useful biomarkers in the diagnosis of acute cerebral infarction and the prediction of stroke severity.

One study has shown that D-dimer levels significantly increase during cerebral infarction and reflect stroke severity ${ }^{22}$. However, we did not find any meaningful increase in D-dimer concentration at the early stage of acute cerebral infarction. Further research is needed to clarify this. 
HSP70 is expressed at low levels in the healthy brain but is induced in all cell types following ischemia, in particular in neurons in the penumbral region ${ }^{21,23)}$. It has been demonstrated that HSP70 and other heat shock proteins are released by stressed cells as free soluble proteins or enclosed within detergent-soluble membrane vesicles ${ }^{13)}$. Therefore, plasma HSP70 levels during the acute stage of cerebral infarction indicate ischemic stroke and predict the size of the penumbral region. Further studies including a large-scale prospective cohort study should be conducted to assess its clinical applicability as a biomarker.

There were several limitations in this study. First, the sample size was small and the number of biomarkers we assessed was limited. In addition, more categories of experimental infarction groups could have been used. Most mice did not survive to conduct a proper neurological examination when MCA occlusion was performed for longer than 1 hour; therefore, we excluded this group and stratified the mice into 30-minute and 60-minute MCAO groups and the evaluation of neurological deficits was based solely on a behavioral assessment. Second, we did quantitative analysis of protein expression with plasma during acute cerebral infarction in this study. To further enhance the diagnostic value, future study will be needed on comparative analysis of expression rates in the four groups of brain tissue and plasma, and also on the differences in gene expression according to infarction volume.

\section{CONCLUSION}

This study used a focal cerebral infarction model to verify S100 $\beta$, MMP-9, and HSP70 as potential biomarkers in the diagnosis of cerebral infarction, estimation of infarction volume, and neurological deficit during the acute stage of the disease. If clinical research continues on to establish useful biomarkers expressed during acute cerebral infarction, there will come a new phase in the treatment of acute cerebral infarction, which will enable faster management of patients and more favorable clinical outcomes.

\section{CONFLICTS OF INTEREST}

No potential conflict of interest relevant to this article was reported.

\section{INFORMED CONSENT}

Informed consent was obtained from all individual participants included in this study.

\section{References}

1. Ageno W, Finazzi S, Steidl L, Biotti MG, Mera V, Melzi D'Eril G, et al. : Plasma measurement of D-dimer levels for the early diagnosis of ischemic stroke subtypes. Arch Intern Med 162 : 2589-2593, 2002

2. Arenillas JF, Alvarez-Sabin J, Molina CA, Chacón P, Fernández-Cadenas I, Ribó $M$, et al. : Progression of symptomatic intracranial large artery atherosclerosis is associated with a proinflammatory state and impaired fibrinolysis. Stroke 39 : 1456-1463, 2008

3. Bader MK, Palmer $S$ : What's the "hyper" in hyperacute stroke? Strategies to improve outcomes in ischemic stroke patients presenting within 6 hours. AACN Adv Crit Care 17 : 194-214, 2006

4. Castellanos M, Leira R, Serena J, Blanco M, Pedraza S, Castillo J, et al. : Plasma cellular-fibronectin concentration predicts hemorrhagic transformation after thrombolytic therapy in acute ischemic stroke. Stroke 35 : 1671-1676, 2004

5. Castillo J, Rodriguez I : Biochemical changes and inflammatory response as markers for brain ischaemia: molecular markers of diagnostic utility and prognosis in human clinical practice. Cerebrovasc Dis 17 Suppl $1: 7-18,2004$

6. Foerch C, Otto B, Singer OC, Neumann-Haefelin T, Yan B, Berkefeld J, et al. : Serum S100B predicts a malignant course of infarction in patients with acute middle cerebral artery occlusion. Stroke 35 : 2160-2164, 2004

7. Foerch $C$, Singer $O C$, Neumann-Haefelin $T$, du Mesnil de Rochemont $R$, Steinmetz $H$, Sitzer M : Evaluation of serum S100B as a surrogate marker for long-term outcome and infarct volume in acute middle cerebral artery infarction. Arch Neurol 62 : 1130-1134, 2005

8. Fujii Y, Takeuchi S, Harada A, Abe H, Sasaki O, Tanaka R : Hemostatic activation in spontaneous intracerebral hemorrhage. Stroke 32 : 883890, 2001

9. Geiger S, Holdenrieder $S$, Stieber P, Hamann GF, Bruening R, Ma J, et al. : Nucleosomes as a new prognostic marker in early cerebral stroke. J Neurol 254 : 617-623, 2007

10. Hacke W, Kaste M, Bluhmki E, Brozman M, Dávalos A, Guidetti D, et al. : Thrombolysis with alteplase 3 to 4.5 hours after acute ischemic stroke. N Engl J Med 359 : 1317-1329, 2008

11. Jiang SX, Lertvorachon J, Hou ST, Konishi Y, Webster J, Mealing G, et al. : Chlortetracycline and demeclocycline inhibit calpains and protect mouse neurons against glutamate toxicity and cerebral ischemia. J Biol Chem $280: 33811-33818,2005$

12. Jin $X$, Xiao C, Tanguav RM, Yang L, Wang F, Chen M, et al. : Correlation of lymphocyte heat shock protein 70 levels with neurologic deficits in elderly patients with cerebral infarction. Am J Med 117 : 406-411, 2004 
13. Joly AL, Wettstein G, Mignot G, Ghiringhelli F, Garrido C : Dual role of heat shock proteins as regulators of apoptosis and innate immunity. J Innate Immun 2 : 238-247, 2010

14. Kleindienst $A$, Ross Bullock $M$ : A critical analysis of the role of the neurotrophic protein $\mathrm{S100B}$ in acute brain injury. J Neurotrauma 23 : 1185-1200, 2006

15. Laskowitz DT, Kasner SE, Saver J, Remmel KS, Jauch EC; BRAIN Study Group : Clinical usefulness of a biomarker-based diagnostic test for acute stroke: the Biomarker Rapid Assessment in Ischemic Injury (BRAIN) study. Stroke 40 : 77-85, 2009

16. Lynch JR, Blessing R, White WD, Grocott HP, Newman MF, Laskowitz DT : Novel diagnostic test for acute stroke. Stroke 35 : 57-63, 2004

17. Maas $M B$, Furie $\mathrm{KL}$ : Molecular biomarkers in stroke diagnosis and prognosis. Biomark Med 3 : 363-383, 2009

18. Rajdev S, Hara K, Kokubo Y, Mestril R, Dillmann W, Weinstein PR, et al. : Mice overexpressing rat heat shock protein 70 are protected against cerebral infarction. Ann Neurol 47 : 782-791, 2000

19. Reynolds MA, Kirchick HJ, Dahlen JR, Anderberg JM, McPherson PH, Nakamura KK, et al. : Early biomarkers of stroke. Clin Chem 49 : 17331739, 2003

20. Rosell A, Ortega-Aznar A, Alvarez-Sabín J, Fernández-Cadenas I, Ribó M, Molina CA, et al. : Increased brain expression of matrix metalloproteinase-9 after ischemic and hemorrhagic human stroke. Stroke 37 : 13991406, 2006

21. Schmidt-Kastner $R$, Zhang B, Belayev $L$, Khoutorova $L$, Amin R, Busto $R$, et al. : DNA microarray analysis of cortical gene expression during early recirculation after focal brain ischemia in rat. Brain Res Mol Brain
Res $108: 81-93,2002$

22. Skoloudik D, Bar M, Sanák D, Bardon P, Roubec M, Langová K, et al. : $D$-dimers increase in acute ischemic stroke patients with the large artery occlusion, but do not depend on the time of artery recanalization. J Thromb Thrombolysis $29:$ 477-482, 2010

23. Snider BJ, Du C, Wei L, Choi DW : Cycloheximide reduces infarct volume when administered up to $6 \mathrm{~h}$ after mild focal ischemia in rats. Brain Res 917 : 147-157, 2001

24. Swanson RA, Morton MT, Tsao-Wu G, Savalos RA, Davidson C, Sharp FR : A semiautomated method for measuring brain infarct volume. J Cereb Blood Flow Metab 10 : 290-293, 1990

25. Whiteley W, Chong WL, Sengupta A, Sandercock P : Blood markers for the prognosis of ischemic stroke: a systematic review. Stroke 40 : e380-e389, 2009

26. Whiteley W, Wardlaw J, Dennis M, Lowe G, Rumley A, Sattar N, et al. : Blood biomarkers for the diagnosis of acute cerebrovascular diseases: a prospective cohort study. Cerebrovasc Dis 32 : 141-147, 2011

27. Wunderlich MT, Wallesch CW, Goertler M : Release of neurobiochemical markers of brain damage is related to the neurovascular status on admission and the site of arterial occlusion in acute ischemic stroke. J Neurol Sci 227 : 49-53, 2004

28. Yenari MA, Giffard RG, Sapolsky RM, Steinberg GK : The neuroprotective potential of heat shock protein 70 (HSP70). Mol Med Today 5 : 525-531, 1999

29. Zhan $X$, Ander BP, Liao IH, Hansen JE, Kim C, Clements D, et al. : Recombinant Fv-Hsp70 protein mediates neuroprotection after focal cerebral ischemia in rats. Stroke 41 : 538-543, 2010 